\title{
Precursor Film in Dynamic Wetting, Electrowetting, and Electro-Elasto-Capillarity
}

\author{
Quanzi Yuan and Ya-Pu Zhao* \\ State Key Laboratory of Nonlinear Mechanics, Institute of Mechanics, Chinese Academy of Sciences, \\ Beijing 100190, People's Republic of China
}

(Received 13 January 2010; published 16 June 2010)

\begin{abstract}
Dynamic wetting and electrowetting are explored using molecular dynamics simulations. The propagation of the precursor film (PF) is fast and obeys the power law with respect to time. Against the former studies, we find the PF is no slip and solidlike. As an important application of the PF, the electro-elastocapillarity, which is a good candidate for drug delivery at the micro- or nanoscale, is simulated and realized for the first time. Our findings may be one of the answers to the Huh-Scriven paradox and expand our knowledge of dynamic wetting and electrowetting.
\end{abstract}

PACS numbers: 68.08.Bc, 05.70.Np, 47.11.Mn

Dynamic wetting is of great importance where physics [1-3], chemistry [4], biology [5], and industry [6,7] intersect. A very thin precursor film (PF), usually a single molecular layer propagating ahead of the nominal contact line, is a crucial part in the wetting and electrowetting process. Hardy's pioneering work [8] predicted the existence of the PF when a droplet spreads. Numerous theoretical $[9,10]$ and experimental $[11,12]$ studies on the spreading droplet have confirmed Hardy's results.

Owing to a variety of applications in micro- or nanofluidic manipulation, lab on chip, micro- or nanolenses, $E$ display, etc., electrowetting, especially electrowetting at a small scale, has attracted considerable attention. In the case of electrowetting, a typical electric energy $\mathbf{E} \cdot \boldsymbol{\mu}(\mathbf{E}$ is the electric field vector and $\boldsymbol{\mu}$ is the dipole moment vector) in the PF cannot be neglected in comparison to the thermal energy $k_{B} T\left(k_{B}\right.$ is the Boltzmann constant and $T$ is the absolute temperature). Polarization is strongest in the PF. Dipolar water molecules may therefore be attracted to the PF. So the PF plays a critical role in electrowetting. But currently there is no report on the effect of the external electric field on the PF to the best of our knowledge.

The properties of the PF have puzzled us for quite a long time. The no-slip boundary in the hydrodynamics theory actually considers the liquid-solid interface, which contains the PF, sticks on the substrate, and is solidlike. Huh and Scriven [13] pointed out theoretically that the no-slip boundary may induce infinite energy dissipation in the region of the contact line and "not even Herakles could sink a solid." Thus the slippage dominates in the PF. Previous studies considered the PF advance adiabatically much faster than the liquid above it. They concluded that the PF behaves diffusively.

In this Letter, we first use molecular dynamics (MD) simulations to explore the atomic details and the transport properties of the PF in dynamic wetting and especially in electrowetting for the first time. Our results show that the PF propagates quickly following the power law in spreading and even faster in electrowetting. But this fast propagation of the PF is owing to continuous and fast diffusing of the surface water molecules to the PF. Against the former studies, the PF itself is not diffusive, but no slip and solidlike and has the lowest mobility in a droplet. From our simulations, the PF may be one of the answers to the Huh-Scriven paradox [13], introducing atomic details to eliminate the infinite energy dissipation due to no slip near the contact line. Then in order to show the important role and applications of the PF as an example, for the first time we realize and simulate the electro-elasto-capillarity of the wrapping and unwrapping of the droplet with graphene due to the unique transport properties of the PF. This process is promising to be a good candidate for drug delivery at the micro- or nanoscale. Our results may help to explain the dynamics of wetting, electrowetting, and electro-elastocapillarity.

MD simulations implemented in LAMMPS [14] were carried out under a constant temperature of $300 \mathrm{~K}$ to explore the properties of the PF on a gold substrate in dynamic wetting and electrowetting. The calculation domain is shown in Fig. 1(a). The extended single point charge water model [15] was used. The gold atoms were modeled as Lennard-Jones particles with $\sigma_{\mathrm{Au}-\mathrm{Au}}=$ $0.2637 \mathrm{~nm}$ and $\varepsilon_{\mathrm{Au}-\mathrm{Au}}=42.5723 \mathrm{~kJ} / \mathrm{mol}$. We specified a hydrogen bond between water molecules if the O-O distance was less than $3.5 \AA$ and simultaneously the angle $\mathrm{O}-\mathrm{H} \cdots \mathrm{O}$ was greater than $100^{\circ}$.

First, the droplet spread on the gold surface and achieved an equilibrium state [Fig. 1(b)], which is in accordance with the experimental configuration [12]. Then, in order to imitate the usual setup of electrowetting, the first layer of the gold surface and the above gold layer were charged with $-1.8--90 e$ and $1.8-90 e$, respectively. $\boldsymbol{E}$ can be calculated $E=Q /\left(A \varepsilon_{0} \varepsilon\right)$ along the $-y$ direction. $Q, A$, $\varepsilon_{0}$, and $\varepsilon$ represent the charges on each layer, the area, the vacuum permittivity, and the relative permittivity of water, respectively. $E$ ranged from $0.0275 \mathrm{~V} / \mathrm{nm}$ to $1.36 \mathrm{~V} / \mathrm{nm}$, which is the typical value for pure water in the electrowetting setup [16]. The wettability of the gold surface increased with the increase of $E$. The simulation time and time step were carefully selected so as to take fully 


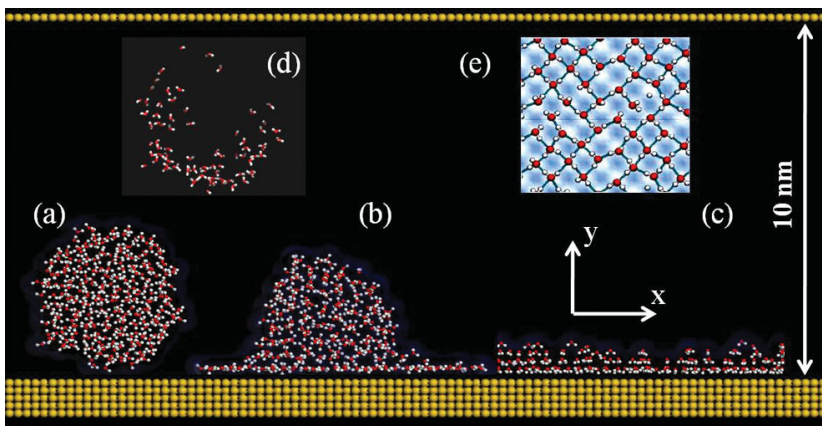

FIG. 1 (color). (a) The initial configuration, which consisted of a gold surface (180 atoms/layer), a cylindrical water droplet (3.5 $\mathrm{nm}$ in diameter), and an above fixed layer of gold atoms (180 atoms). Red, white, and yellow atoms represent oxygen, hydrogen, and gold atoms, respectively. The distance between the two gold layers is $10.0 \mathrm{~nm}$. (b) The configuration after the full spreading of the droplet. (c) Complete wetting of the droplet. (d) The water molecules in the initial configuration (a), which finally formed the PF in (b). (e) The snapshot of the twodimensional $H$-bond network in the PF. The blue lines represent $H$ bonds. And the background color illustrates the potential surface of the gold substrate.

into account the behavior of the droplet and the atomic details.

As shown in Fig. 2(a), we found the propagation of the PF (about $3 \AA$ in height) was fast and obeyed the power law of $R \sim t^{0.1554}$ in spreading, which is supported by experiments of about $R \sim t^{1 / 7}$ [17]. For instance, we adopted the molecular kinetic theory, which was proposed by Erying et al. [18], to explain the physical mechanism behind these phenomena. For the water molecules' jump between gold surface sites separated by a distance $\lambda$ with a frequency $K_{0}$, $v=2 K_{0} \lambda \sinh \left(w / 2 n k_{B} T\right)$, where $v$ is the advancing velocity, $w$ is the work per unit area done by the driving force, and $n$ is the density of sites on the solid gold surface. Blake and Haynes [19] took $w=\gamma\left(\cos \theta_{0}-\cos \theta\right)$ for a spreading droplet without considering the PF, where $\gamma, \theta_{0}$, and $\theta$ represent surface tension, static, and dynamic contact

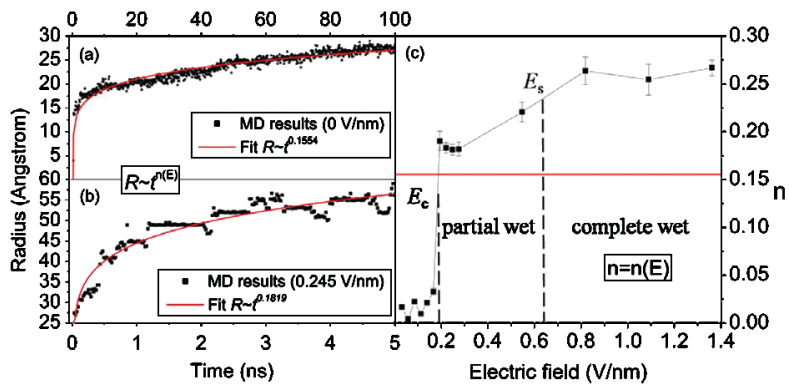

FIG. 2 (color). The propagation of the radius $R$ of the PF with respect to the time $t$. (a) The spreading of the droplet. (b) The electrowetting of the droplet when the external electric field $E=$ $0.245 \mathrm{~V} / \mathrm{nm}$. (c) The change of $n$ with respect to $E$. The red line is $n=0.1554$ when the droplet spread. The left and right dashed lines represent the critical electric field $E_{c}$ and the saturated field $E_{s}$ in electrowetting, respectively. angle, respectively. And they concluded when $\theta \rightarrow 0, R \sim$ $t^{1 / 5}$ for a two-dimensional (2D) cylindrical droplet. But when the droplet is small, $w$ becomes complicated. Here, following Derjaguin and Churaev [20], the driving work of the spreading droplet considering the PF can be written as $w=w_{V}+w_{P}+w_{S}$, where $w_{V}$ is the work per unit area arising from van der Waals interactions, $w_{P}$ is from the polar interactions between water molecules, and $w_{S}$ is from the different structure of the PF from the bulk liquid. In the case of electrowetting, an additional average electric energy $w_{E}$ describing the interaction between $\mathbf{E}$ and $\boldsymbol{\mu}_{\mathbf{i}}$ is $w_{E} \sim \sum_{i}\left[-|\mathbf{E}|\left|\boldsymbol{\mu}_{i}\right| L\left(|\mathbf{E}|\left|\boldsymbol{\mu}_{i}\right| / k_{B} T\right)\right]$, where $L(x)$ is the Langevin function. Thus, the governing equation for spreading and electrowetting can be written as

$$
v=2 K_{0} \lambda \sinh \left(\frac{w_{V}+w_{P}+w_{S}+w_{E}}{2 n k_{B} T}\right)
$$

For $w_{V}+w_{P}+w_{S}+w_{E} \ll n k_{B} T, \quad v \sim\left(w_{V}+w_{C}+\right.$ $\left.w_{S}+w_{E}\right) / s_{0}$, where $\varsigma_{0}=n k_{B} T / K_{0} \lambda$ represents a friction coefficient per unit length. This equation is too complicated to have an analytical solution. So we used the power law $R \sim t^{n(E)}$ [21] to fit the relationship between $R$ and $t$. The power law $R \sim t^{0.1819}$ fitted well to the MD data when $E=0.245 \mathrm{~V} / \mathrm{nm}$ in Fig. 2(b). The steps in Fig. 2(b) were caused by the relaxation time ( $\sim 1 \mathrm{~ns})$ of water dipoles to $E$. From the relationship between $n(E)$ and $E$ as shown in Fig. 2(c), a critical electric field $E_{c}$ (about $0.175 \mathrm{~V} / \mathrm{nm}$ ) and a saturated field $E_{s}$ (about $0.625 \mathrm{~V} / \mathrm{nm}$ ) were found in accordance with the experimental observation [16].

The average of the local density profile around a water molecule was plotted in Fig. 3. The first peak for the bulk water in spreading (black line) was about $2.675 \AA$, which is the average intermolecular distance between water molecules $d_{w}$. The $d_{w}=2.715 \AA$ of the PF in spreading (red line) was a little larger than the $d_{w}$ of the bulk water. The $d_{w}$ of the PF increased with the increase of $E$. When $E=$ $1.36 \mathrm{~V} / \mathrm{nm}, d_{w}$ increased to $2.985 \AA$.

From the above discussion, the advancing of the PF was found to be fast. But does it mean that the PF propagated

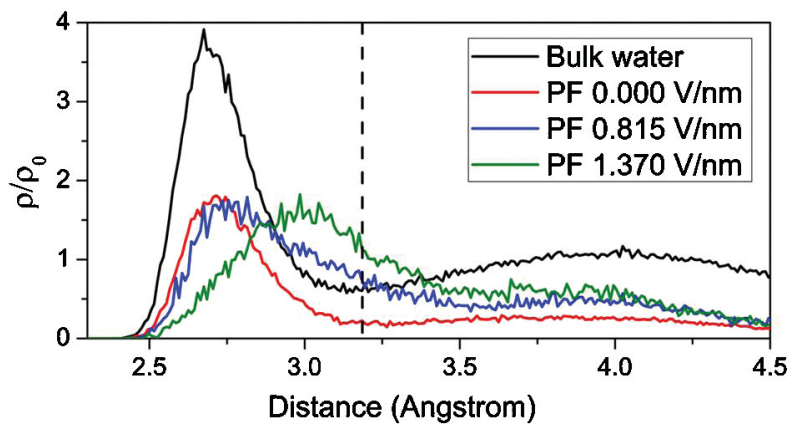

FIG. 3 (color). The average of the local density profile $\left(\rho / \rho_{0}\right.$, $\rho_{0}=1000 \mathrm{~kg} / \mathrm{m}^{3}$ ) around a water molecule. The first peak of each line represents the average intermolecular distance between water molecules $d_{w}$. The dash line represents the average intermolecular distance between gold atoms $d_{g}$ in the substrate. 
like liquid and slipped on the gold surface to eliminate the singularity? The answer is negative. Two issues are raised. First, we observed that the molecules, which finally formed the PF, came almost from the surface in the initial state of the droplet [Fig. 1(d)]. If the PF slips to expand, it is hard to imagine the water molecules, which finally composed the $\mathrm{PF}$, were not mainly from the bulk. Second, if the PF propagated as liquid, $d_{w}$ in the PF should decrease to bear more pressure.

To address these issues, we tracked each water molecule in spreading, and took some of the path lines to get Fig. 4. If the path line assembles, it means that this molecule diffused slowly. If the path line disperses, it means that this molecule diffused quickly. The droplet was divided into 5 regions. (a) The bulk water molecules (grey) did a random walk due to $k_{B} T$. (b) The surface molecules (pink) had the highest mobility. Bearing less restriction than in the bulk, they moved quickly in the surface. (c) The water molecules in the nominal contact region (orange) also moved very fast, but were confined in this region. It can be seen in the left orange region that there may exist vortices, which need further study. (d) The water molecules in the PF (blue) had the lowest mobility. The assembled path lines indicated that the PF molecules vibrated around one site with a amplitude less than $3 \AA$ and hardly went away and had few exchanges with other parts. (e) Some water molecules (purple) moved very fast at the surface. But once diffusing to the region of the PF, they were pinned by the gold surface and got a quick damping. Then these molecules acted as PF molecules with the lowest mobility. Because of the surface molecules' continuous and fast diffusing to the PF, the PF propagates fast without high energy dissipation. The path lines in electrowetting validated this conclusion again. There are frequent molecular exchanges among the surface, bulk, and nominal contact region. Here, in order to show the mobility clearly, these exchanges are not shown in Fig. 4.

To quantify our observation, we evaluated the transport properties by computing the self-diffusion coefficient $D$ of water molecules in spreading and electrowetting, respectively. Since the droplet was cylindrical in our simulations,

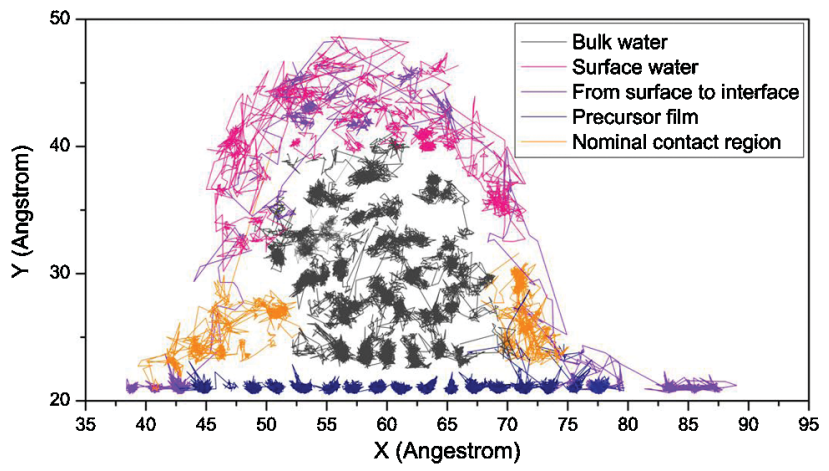

FIG. 4 (color). The path lines of water molecules belonging to different regions. we consider it to be a $2 \mathrm{D}$ problem and considered only water diffusion in the $x$ and $y$ direction. $D$ of water is related to the slope of the water mean-squared displacement by the Einstein relation: $D=\frac{1}{4} \lim _{t \rightarrow \infty}[\langle| \vec{r}(t)-$ $\left.\left.\left.\vec{r}(0)\right|^{2}\right\rangle / \Delta t\right]$, where $|\vec{r}(t)-\vec{r}(0)|$ is the distance traveled by molecule $i$ over some time interval of length $\Delta t$, and the squared magnitude of this value is averaged over many such time intervals. The results are listed in Table I. When the droplet spread, $D=1.132 \times 10^{-5} \mathrm{~cm}^{2} / \mathrm{s}$ in the PF $(20 \leq y \leq 22$, in Fig. 4), which was $50.4 \%$ of $D=$ $2.246 \times 10^{-5} \mathrm{~cm}^{2} / \mathrm{s}$ of bulk water. $D$ of the surface region and nominal contact region are $7.354 \times 10^{-5} \mathrm{~cm}^{2} / \mathrm{s}$ and $5.434 \times 10^{-5} \mathrm{~cm}^{2} / \mathrm{s}$, respectively. When the external electric field was imposed, $D$ decreased to the orders of $10^{-6} \mathrm{~cm}^{2} / \mathrm{s}$, about $6 \%$ of $D$ of bulk water. These results indicate that the PF actually acts solidlike.

From the above discussion, the earlier-mentioned two issues can be addressed here. First, does the PF slip to advance? The continuous and fast diffusing of surface water molecules to the front of the PF allows for the fast propagation of the PF. Second, why does $d_{w}$ in the PF increase with the increase of $E$ ? The water molecules in the $\mathrm{PF}$ are attracted by gold substrate through van der Waals force in spreading, and through van der Waals force and electrostatic force in electrowetting. We can find in Fig. 1(e) that the water molecules in the PF preferred to stay in the potential well between gold atoms to reduce the interfacial energy. The average intermolecular distance between gold atoms $d_{g}$ is $3.195 \AA$ A larger than $d_{w}$, which is responsible for the increase of $d_{w}$.

The unique hydrogen bonds ( $H$ bonds) network in the $\mathrm{PF}$ allows for the unique behavior of the PF. So we computed the average number of $H$ bonds formed by (1) a water molecule in the PF with neighboring water molecules also in the PF $\left(N_{\mathrm{in}-\mathrm{PF}}\right),(2)$ a water molecule in the PF with water molecules above the PF $\left(N_{\mathrm{PF}-\mathrm{Bulk}}\right)$, and (3) a water molecule in the PF with any other water molecules $\left(N_{t \mathrm{PF}}\right)$ (Table II). $N_{\text {in-PF }}$ was found to be $89.8 \% \pm 7.4 \%$ of $N_{t \mathrm{PF}}$, which means that a 2D $H$-bond network was formed in the PF [Fig. 1(e)] and is also validated by experiments [22]. And $N_{\mathrm{PF}-\mathrm{Bulk}}$ was $17.67 \%$ of $N_{t \mathrm{PF}}$, which means there were a few dangling $\mathrm{OH}$ bonds. The $2 \mathrm{D} H$-bond network between water molecules in the PF dominated the behaviors of water molecules in the PF, and made the diffusion of molecules above the PF easier.

To further explore the properties of the PF and show the important role of the PF as an example, an electro-elastocapillary process was simulated for the first time. First we used graphene (its flexural stiffness $B$ is about $0.2 \mathrm{nN} \cdot \mathrm{nm}$ [23]) to wrap the droplet. Since the radius of the droplet is

TABLE I. The change of the self-diffusion coefficient $D$ of the PF molecules with respect to the electric field $E$.

\begin{tabular}{lllllll}
\hline \hline$E(\mathrm{~V} / \mathrm{nm})$ & 0.0000 & 0.0272 & 0.0543 & 0.0815 & 0.1086 & 0.1358 \\
$D\left(10^{-5} \mathrm{~cm}^{2} / \mathrm{s}\right)$ & 1.1320 & 0.1359 & 0.1360 & 0.1536 & 0.1405 & 0.1547 \\
\hline \hline
\end{tabular}


TABLE II. The change of the average number of $H$ bonds with respect to the electric field $E$.

\begin{tabular}{lllllll}
\hline \hline$E(\mathrm{~V} / \mathrm{nm})$ & 0.0000 & 0.0272 & 0.0543 & 0.0815 & 0.1086 & 0.1358 \\
$N_{\text {in-PF }}{ }^{\mathrm{a}}$ & 1.9598 & 1.9568 & 1.7706 & 1.7610 & 1.6512 & 1.6230 \\
$N_{\mathrm{PF}-B u l k}{ }^{\mathrm{b}}$ & 0.4205 & 0.3510 & 0.3528 & 0.3580 & 0.2788 & 0.2800 \\
$N_{t \mathrm{PF}}{ }^{2}$ & 2.3803 & 2.3078 & 2.1234 & 2.1190 & 1.9300 & 1.9030 \\
\hline \hline
\end{tabular}

${ }^{\mathrm{a}} N_{\mathrm{in}-\mathrm{PF}}$ is formed by a water molecule in the PF with neighboring water molecules also in the PF.

${ }^{\mathrm{b}} N_{\text {PF-Bulk }}$ is formed by a water molecule in the PF with water molecules above the PF

${ }^{\mathrm{c}} N_{t \mathrm{PF}}$ is formed by a water molecule in the PF with any other water molecules.

larger than the elasto-capillary length $L_{\mathrm{EC}}=(B / \gamma)^{1 / 2}$ [24], the graphene automatically wraps the droplet. Then this wrapped droplet unwrapped on the gold substrate under $E=0.544 \mathrm{~V} / \mathrm{nm}$ along the $-y$ direction as shown in Fig. 5. The van der Waals force between the graphene and the substrate was weakened to $0.1 \%$ to avoid interfering with the process. Because the propagation of the PF is faster than the liquid above it, and because of the solidlike property of the $\mathrm{PF}$, the $\mathrm{PF}$ pushed the graphene to unwrap with a force of the order of $1 \mathrm{nN} / \mathrm{nm}$ from our calculations. So using the unique transport properties of the PF, the electro-elasto-capillarity can be realized and used for the drug delivery at a micro- or nanoscale.

In conclusion, we performed MD simulations to explore the atomic details and the transport properties of the PF in dynamic wetting, especially electrowetting and the electroelasto-capillarity for the first time. We found the advancing of the PF was fast in spreading and even faster in electrowetting. The propagation of $R$ to $t$ fitted the power law $R \sim$ $t^{n(E)}$ very well. The composition of the PF and the calculation of $d_{w}$ in the PF indicated that the PF might not be diffusive, and did not slip on the substrate. The path lines and self-diffusion coefficient of water molecules confirmed our point that the PF acted solidlike, and was pinned by the

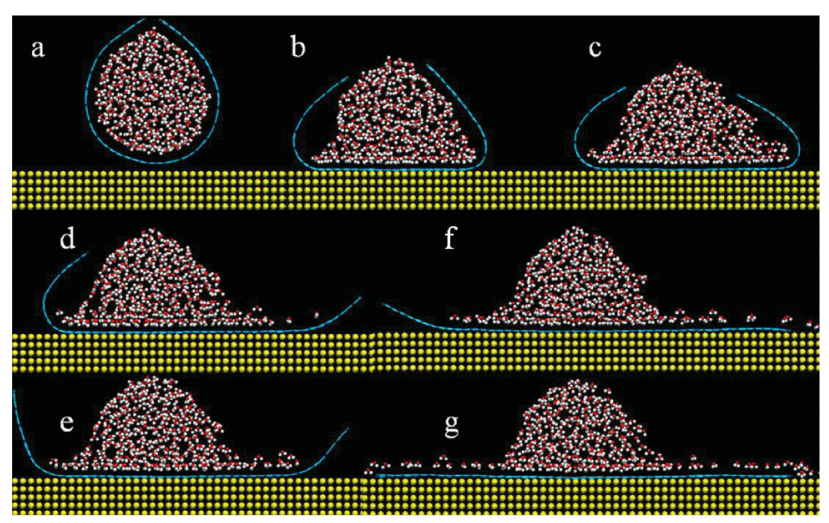

FIG. 5 (color). The dynamic electro-elasto-capillary process of a droplet on gold substrate. The wrapped droplet unwraps on the gold substrate under $E=0.544 \mathrm{~V} / \mathrm{nm}$ along the $-y$ direction. The blue line represents the graphene. The red, white, and yellow atoms represent oxygen, hydrogen, and gold atoms, respectively. substrate. The 2D $H$-bond network allowed for the unique transport properties of the PF. From our simulations, the PF may be one of the answers to the Huh-Scriven paradox, introducing atomic details to eliminate the infinite dissipation due to no slip near the contact line. Also, an electroelasto-capillary process was simulated as an example to show the importance and applications of the PF. The PF pushed the graphene to unwrap with a total force of the order of $1 \mathrm{nN} / \mathrm{nm}$. Using the unique transport properties of the PF, the dynamic process of wrapping and unwrapping of a droplet is promising to be a good candidate for the drug delivery at a micro- or nanoscale. It is expected that our finding will help to expand our knowledge of dynamic wetting, electrowetting, and electro-elasto-capillarity.

This work was jointly supported by the National HighTech R\&D Program of China (863 Program, Grants No. 2007AA04Z348 and No. 2007AA021803), National Basic Research Program of China (973 Program, Grant No. 2007CB310500), and National Natural Science Foundation of China (NSFC, Grants No. 10772180 and No. 10721202).

*Corresponding author. yzhao@imech.ac.cn (Ya-Pu Zhao)

[1] D. Bonn et al., Rev. Mod. Phys. 81, 739 (2009).

[2] C. L. Wang et al., Phys. Rev. Lett. 103, 137801 (2009).

[3] J. De Coninck and T. D. Blake, Annu. Rev. Mater. Res. 38, 1 (2008).

[4] Q.Z. Yuan and Y.P. Zhao, J. Am. Chem. Soc. 131, 6374 (2009).

[5] R. Zhou et al., Science 305, 1605 (2004).

[6] A. Noy et al., Nano Today 2, 22 (2007).

[7] M. Whitby and N. Quirke, Nature Nanotech. 2, 87 (2007).

[8] W. B. Hardy, Philos. Mag. 38, 49 (1919).

[9] D. B. Abraham et al., Phys. Rev. Lett. 65, 195 (1990).

[10] P. De Gennes, Rev. Mod. Phys. 57, 827 (1985).

[11] D. Ausserré, A. M. Picard, and L. Léger, Phys. Rev. Lett. 57, 2671 (1986).

[12] H. P. Kavehpour, B. Ovryn, and G. H. McKinley, Phys. Rev. Lett. 91, 196104 (2003).

[13] C. Huh and L. Scriven, J. Colloid Interface Sci. 35, 85 (1971).

[14] S. Plimpton, J. Comput. Phys. 117, 1 (1995).

[15] H. J.C. Berendsen, J.R. Grigera, and T.P. Straatsma, J. Phys. Chem. 91, 6269 (1987).

[16] F. Mugele and J. Baret, J. Phys. Condens. Matter 17, R705 (2005).

[17] G. McHale et al., J. Phys. D 28, 1925 (1995).

[18] S. Gladstone, K. Laidler, and H. Eyring, The Theory of Rate Processes (Princeton University, New York, 1941).

[19] T. D. Blake and J. M. Haynes, J. Colloid Interface Sci. 30, 421 (1969).

[20] B. Derjaguin and N. Churaev, J. Colloid Interface Sci. 49, 249 (1974).

[21] L. Tanner, J. Phys. D 12, 1473 (1979).

[22] A. Mugarza et al., J. Phys. Chem. C 112, 14052 (2008).

[23] N. Patra, B. Wang, and P. Král, Nano Lett. 9, 3766 (2009).

[24] C. Py et al., Phys. Rev. Lett. 98, 156103 (2007). 